\title{
The importance of establishing supportive supervision feedback audit system in Gurage Zone health department
}

\author{
Habtamu Mergia ${ }^{1}$, Kassahun Trueha Dumga ${ }^{2 *}$
}

\begin{abstract}
${ }^{1}$ Technical Advisor for Management Systems, Yale Global Leadership Initiative, ${ }^{2}$ Department of Statistics, Wolkite University, Wolkite, Ethiopia
\end{abstract}

Received: 03 January 2020

Revised: 14 February 2020

Accepted: 15 February 2020

\section{*Correspondence:}

Dr. Kassahun Trueha Dumga,

E-mail: ktktantos@gmail.com

Copyright: (c) the author(s), publisher and licensee Medip Academy. This is an open-access article distributed under the terms of the Creative Commons Attribution Non-Commercial License, which permits unrestricted non-commercial use, distribution, and reproduction in any medium, provided the original work is properly cited.

\begin{abstract}
Supportive supervision is one of the most important tasks in health systems management. A baseline assessment conducted by the primary health care transformation initiative had shown that the Gurage Zone health department had been conducting regular supportive supervision every quarter for the last five years in Gurage Zone. The study proposed establishing a feedback auditing system as an intervention to routinely assess the progress of previous visit actions included in the written feedback. The finding showed the culture of working on previous supportive supervision action items by woreda (district) health offices were significantly improved.
\end{abstract}

Keywords: Feedback audit, $\mathrm{M}$ and E, Supportive supervision

\section{INTRODUCTION}

Supportive supervision is a facilitative approach used for continuous improvements in the quality of care in health care centers. WHO defines supportive supervision as" helping to make things work, rather than checking to see what is wrong". 'Supportive approach is mostly linked to an increase health workers motivation and job persormance. $^{2}$ The traditional approach was based on the thinking that health workers are unmotivated and need strong outside control to perform correctly and directive and authoritarian, but this approach limits the performance of basic supervision tasks and demoralizes health workers. ${ }^{3}$

Supportive supervision is a quality result oriented and aims at improving individual's performance, Marshall and Fehringer noted that supportive supervision improves different part of the monitoring and evaluation process, like data collation and motivation of health workers. ${ }^{4}$ Since supportive supervision is carried out in a respectful and non- controlling manner with a focus on supportive visits as an opportunity to improve knowledge and skills for the health workers. As human behavior the health workers will consider themselves important when the supervision is conducted as a form of supportive way. Supportive supervision can also benefit the overall system by improving the quality of health care and the performance of health care providers. ${ }^{5,6}$

Different organizations are advocating the inclusion of supportive supervision in $\mathrm{M}$ and $\mathrm{E}$, because it improves monitoring problems by making smooth communication between supervisors and supervisees, and also it is a type ongoing monitoring method. ${ }^{7,8}$ In 2009, UNAIDS developed supportive supervision and data auditing tool for $\mathrm{M}$ and $\mathrm{E}$ in order to improve data quality, and strengthen local $\mathrm{M}$ and $\mathrm{E}$ capacity. ${ }^{9}$ To support this 
initiative and to reach the society, researches are needed. The purpose of this study was to develop case studies of supportive supervision projects that could be used as examples for other programs wishing to use supportive supervision in $\mathrm{M}$ and $\mathrm{E}$.

\section{BACKGROUND OF THE STUDY AREA}

Gurage Zone has 13 woredas (districts) and two city administration that provides health services to a total population of 1,686,741. Gurage Zone health department is providing administrative and technical support for 15 Woreda health offices, one general hospital, 3 primary hospitals and 71 health centers to deliver quality health service for the community. Integrated supportive supervision is a technical support conducted by higher level institutions with the objective to review the performances, process and service standards of lower level institutions and provide support to fill the existing gaps and challenges to deliver quality health services. Integrated supportive supervision is an approach used to provide technical support for many years and already institutionalized in the system of Ethiopian health sector.

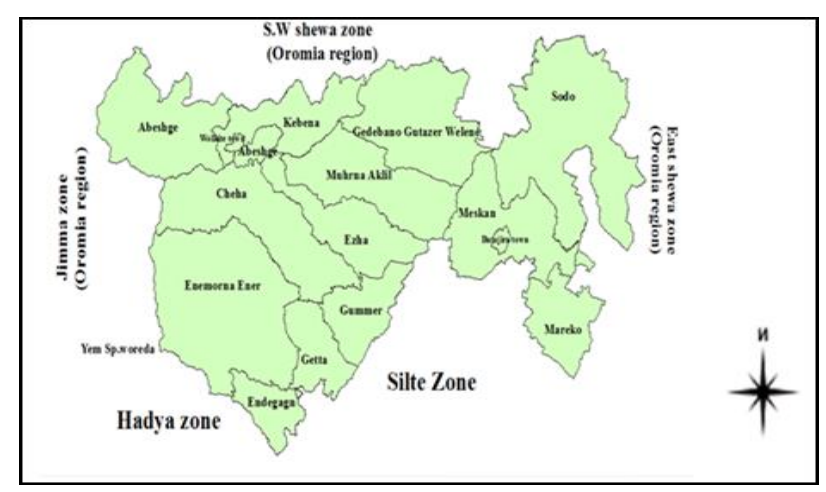

Figure 1: Study area.

A baseline assessment conducted by primary health care transformation initiative (PTI) project in collaboration with Gurage zone health department using management standard indicated that Gurage zone health department had been conducting regular supportive supervision on a quarterly basis for the last five years. Past practices indicated Gurage zone health department established supportive supervision setting that includes planning for supervision, developing supervision checklist and providing orientation for all staffs on the checklist before departing for supervision. During the supervision supervisors list out action items for improvement with woreda health office management for action. The supervision team summarized key finding by program areas and provide official written feedback for woreda health offices and health facilities.

Zonal health department had no means to track the progress of previous supervision visit action items. Due to this ZHD had no information about which woredas have worked on the action items and improved the gaps identified during supportive supervision. ZHD management and staffs doubt the improvement of Woreda Health Offices due to supportive supervision, while ZHD is providing adequate and regular supportive supervision.

\section{INTERVENTION}

In June, 2018 Gurage zone health department management and PTI technical advisor reviewed the approaches used in integrated supportive supervision and improvement in performance of woreda health offices because of working on supportive supervision agreed action items. The team found that the integrated supervision approach used and the support provided was according to the standard but attention was not given to follow the progress of action items. The team proposed establishing feedback auditing system as an intervention to routinely assess the progress of previous visit action actions included in the written feedbacks. Questions to address the progress of previous visit action items included in integrated supportive supervision checklist. In all visits, before starting current visit agendas included in the checklist the supervision team is expected to check the status previous visit action items.

Feedback improvement rate (FIR) is designed as an indicator to quantify the improvement of identified gaps of last visits by woreda health offices and health facilities. Feedback improvement rate is calculated as the number of action items improved from previous feedback to the number of previous action items included in the supervision feedback.

In addition to measuring the improvement of previous actions items, Gurage zone health department used feedback improvement rate as one indicator to know how Woreda health offices and health centers are using the supervision finding as input to improve management standards and work to deliver quality health service for the community. Gurage zone health department used feedback improvement rate as one indicator to measure the performance of Woreda health offices management.

\section{OUTCOMES}

Gurage zone health department started routine feedback audit by making one major agenda during supportive supervision visit. The finding of integrated supportive supervision conducted in February, 2019 and August, 2019 showed the culture of working on previous supportive supervision action items by woreda health offices was significantly improved, which is similar result as other research. ${ }^{10}$ Feedback audit conducted by Gurage zone health department in August, 2019 showed the average feedback improvement rate of woreda health offices improved from $47 \%$ to $77 \%$. The following graph shows feedback improvement rate of Woreda Health Offices in Gurage zone. 


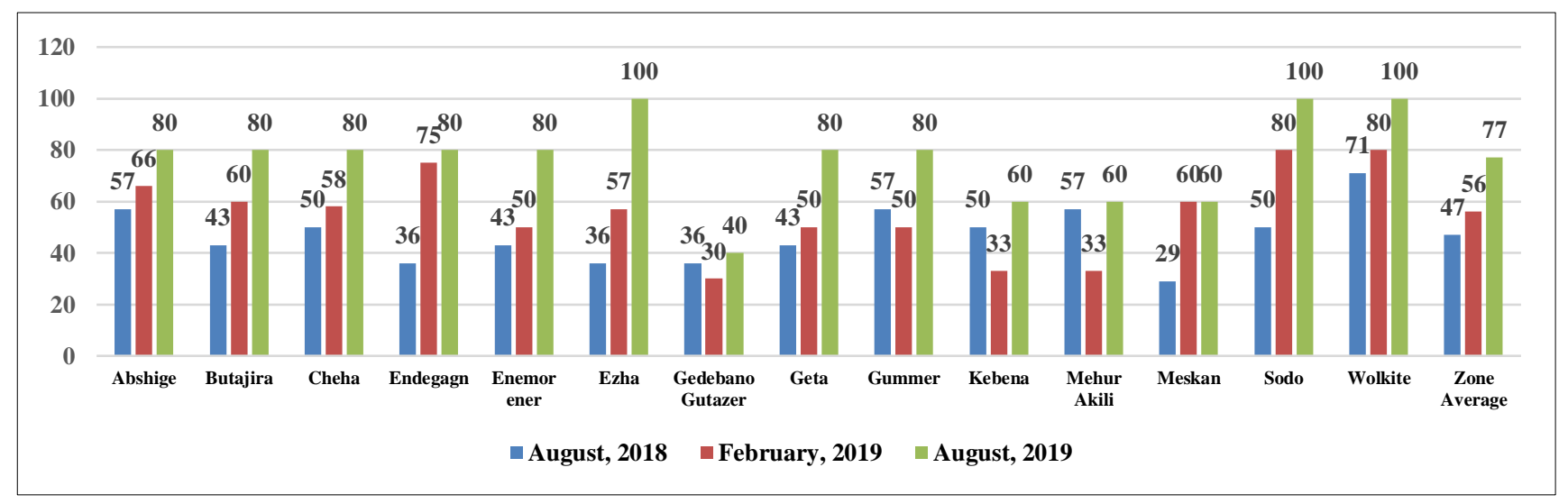

Figure 2: Woredas feed improvement rate.

\section{CONCLUSION}

Conducting regular supportive supervision and providing feedback only is not enough to bring performance improvement by Woreda Health Offices. Designing and using feedback audit system and following the status of action items will make the supervision cycle complete and maximize the output of the supportive supervision.

The finding also showed that, woreda health offices with high feedback improvement rate have high health service performance. Example; Sodo and Ezha woreda health offices have $100 \%$ feedback improvement rate and they are the first and second high performing woredas in the zone respectively.

\section{Recommendations}

In addition to conducting regular supportive supervision, establishing follow up mechanism to know the status of previous visit action points is helpful to maximize the rate of improvement in health system.

\section{Funding: No funding sources}

Conflict of interest: None declared

Ethical approval: Not required

\section{REFERENCES}

1. WHO, Training for mid-level managers (MLM) Module 4: Supportive supervision, The Department of Immunization, Vaccines and Biological; 2008.

2. Bailey C, Blake C, Schriver M, Cubaka VK, Thomas T, Martin Hilber A. A systematic review of supportive supervision as a strategy to improve primary healthcare services in Sub-Saharan Africa. Int J Gynecol Obstet. 2016;132(1):117-25.

3. Marquez L, Kean L. Making supervision supportive and sustainable: new approaches to old problems [maximizing access and quality initiative, MAQ paper no. 4]. Washington, DC: U.S. Agency for International Development; 2002.

4. Marshall A, Fehringer J. A Case Study from Haiti Supportive Supervision in Monitoring and Evaluation with Community Based Health Staff in HIV Programs; 2013.

5. McAuliffe E, Daly M, Kamwendo F, Masanja H, Sidat $\mathrm{M}$, de Pinho $\mathrm{H}$. The critical role of supervision in retaining staff in obstetric services: a three country study. PloS one. $2013 ; 8: 3$.

6. Kyabayinze DJ, Asiimwe C, Nakanjoko D, Nabakooza $\mathrm{J}$, Counihan H, Tibenderana JK. Use of RDTs to improve malaria diagnosis and fever case management at primary health care facilities in Uganda. Malar J. 2010;9:200.

7. Marquez L, Kean L. Making supervision supportive and sustainable: New approaches to old problems. Maximizing Access and Quality paper no. 4. 2002. Available at: http://www.popline.org/node/265419. Accessed on 24 December 2019.

8. Marquez L, Kean L. Making supervision supportive and sustainable: new approaches to old problems, maximizing access and quality initiative, 2002. Available at: https://www.k4health.org/sites/default/ files/maqpaperonsupervision.pdf. Accessed on 25 December 2019.

9. Joint United Nations Programme on HIV/AIDS (UNAIDS). 12 Components Monitoring \& Evaluation System Assessment: Guidelines to Support Preparation, Implementation, and Follow-up Activities. Geneva, Switzerland: UNAIDS; 2010. Available at: http://www.unaids.org/en/media/unaids/contentassets/d Accessed on 24 December 2019.

10. Mwendwa P, McAuliffe E, Uduma O, Masanja H, Mollel H. The Impact of Supportive Supervision on the Implementation of HRM processes; A Mixed-Methods study in Tanzania. Health Syst Policy Res. 2017;4:1.

Cite this article as: Mergia H, Dumga KT. The importance of establishing supportive supervision feedback audit system in Gurage Zone health department. Int J Community Med Public Health 2020;7:1194-6. 